\title{
Constrictive pericarditis presenting with massive ascites without hemodynamic echocardiographic features
}

\author{
Hasan Ashraf* \\ Department of Internal Medicine, Indiana University, Indiana, USA
}

Received: December 1, 2016

DOI: $10.5430 /$ crim.v4n1p54
Accepted: December 20, 2016 Online Published: January 13, 2017

URL: https://doi.org/10.5430/crim.v4n1p54

\begin{abstract}
A 27-year-old woman presented to the hospital because of a five-month-history of rapidly-accumulating ascites, dyspnea, and fatigue. The patient was otherwise asymptomatic, and required repeated large volume paracenteses. Physical exam was benign except for hepatomegaly and abdominal distension. Laboratory testing demonstrated elevation of transaminases, but further testing was all negative. A chest CT showed pericardial thickening. Subsequent echocardiography was performed to evaluate for constrictive pericarditis, but apart from inferior vena cava (IVC) dilation, there were no other findings suggestive of pericardial constriction. A subsequent cardiac catheterization was suggestive of constrictive pericarditis, so the patient underwent a pericardiectomy. The Mayo Clinic echocardiography diagnostic criteria presents a diagnostic paradigm where the presence of mitral inflow E/A $>0.8$ and the presence of a dilated IVC concomitantly provide good sensitivity for echocardiographic diagnosis of constrictive pericarditis (CP). Due to the good sensitivity and specificity of echocardiographic findings, the lack of any characteristic finding is surprising, and suggests the importance of other diagnostic modalities such as CT, cardiac MRI, and cardiac catheterization in conjunction with echocardiography when there is a high suspicion for CP.
\end{abstract}

Key Words: Constrictive pericarditis, Ascites, Echocardiography

\section{INTRODUCTION}

Diagnosis of constrictive pericarditis is challenging because of its symptomatology and hemodynamic characteristics are similar to those of other pathologies. Correct diagnosis involves a broad approach utilizing clinical history, physical examination, and imaging, involving echocardiography. A high clinical suspicion should be present for correct diagnosis since echocardiography alone can present without typical hemodynamic findings of constrictive pericarditis $(\mathrm{CP})$.

\section{Case presentation}

A 27-year-old woman with a one-year prior history of acute pericarditis complicated by pericardial effusion presented to the hospital with a main complaint of rapidly accumulating ascites which had started five months prior. Her abdominal distension had gradually progressed and an ultrasound diagnosed large volume ascites. She was sent to a hospital for a paracentesis, and since then she had 10 paracenteses. Apart from mild abdominal pain associated the distension that would resolve upon drainage of ascitic fluid, she was asymptomatic. The patient had normal vital signs on examination; physical examination was negative for jugular venous distension, no inspiratory rise in venous pressure (Kussmaul's sign), or pericardial knock. Hepatomegaly was present without pulsatility, as well as large volume ascites and trace pitting lower extremity edema; heart sounds were

*Correspondence: Hasan Ashraf; Email: ashrafh@iu.edu; Address: Department of Internal Medicine, Indiana University, Indiana, USA. 
normal. The patient's home medications included citalopram, tramadol, spironolactone, and an estradiol patch. Of note, her acute pericarditis had been treated with steroids after failure of colchicine therapy, and the symptoms had henceforth resolve post-steroid usage. A 12-lead electrocardiogram showed a normal sinus rhythm with poor $\mathrm{R}$ wave progression and low voltage. BNP was mildly elevated $(121 \mathrm{pg} / \mathrm{ml})$. Transaminases were elevated with a mixed hepatic and cholestatic pattern. All hepatic testing, including hepatoviridae serologies, ceruloplasmin, autoimmune antibodies, and A1AT were unremarkable. A chest CT demonstrated pericardial thickening to $5 \mathrm{~mm}$ with moderate amount of pericardial effusion. A transjugular liver biopsy was performed which demonstrated elevated pulmonary capillary wedged pressure. Biopsy showed mild portal chronic inflammation. An echocardiogram was performed which showed a dilated IVC, but with a preserved EF of $53 \%$ and no relaxation pattern abnormalities. None of the characteristic echo findings for constrictive pericarditis were present except for the dilated IVC. A left and right heart catheterization was performed which showed elevated and nearly equal right and left heart filling pressures with prominent Y-descent and "dip-and-plateau" pattern all consistent with constrictive physiology. The patient was transferred to the cardiac surgery service where she underwent a pericardiectomy. Intraoperatively, the pericardium was found to be hard and densely adherent to the heart; the pericardium was resected from its pleural investment and the patient immediately had a drop in the CVP from 20 to 2, confirming the diagnosis of constrictive pericarditis. The patient has done well post-operatively and no longer has ascites.

\section{Discussion}

$\mathrm{CP}$ is an inflammatory process involving the pericardium eventually leading to fibrosis and subsequent impaired diastolic cardiac filling. The etiology is diverse, varies geographically, and has evolved over time. Historically, iatrogenic causes were rare but have become significantly more common with the increase in cardiac procedures. In developed countries, the most common etiology apart from cardiac surgery is an idiopathic form of pericarditis that is usually assumed to have been secondary to a viral infection. In developing countries, by far the most common etiology is tuberculosis, and this incidence can rise to $80 \%$ in some parts of sub-Saharan Africa. ${ }^{[1]}$

Diagnosis of constrictive pericarditis is often challenging, requiring both clinical suspicion based on presentation with electrocardiography, radiographic imaging and echocardiographic and cardiac catheterization findings. There are variant "gold standards" for the diagnosis and no consensus on

Published by Sciedu Press what suffices for diagnosis. Echocardiographic testing in all suspected pericardial conditions is recommended by the American College of Cardiology/American Heart Association. ${ }^{[2]}$ The cardinal echocardiographic findings are based mainly on the hemodynamic physiological attributes of $\mathrm{CP}$, with its impaired ventricular filling of the left ventricle due to the volume limitations placed by the thickened pericardium. These findings are present on both two dimensional and M-mode echocardiography as well as Doppler echocardiography: Some of the common $\mathrm{M}$-mode findings include abrupt posterior motion of the ventricular septum (septal shudder and bounce); lack of systemic venous return with inspiration; and notching of the ventricular septal tracing in early diastole. ${ }^{[3,4]}$

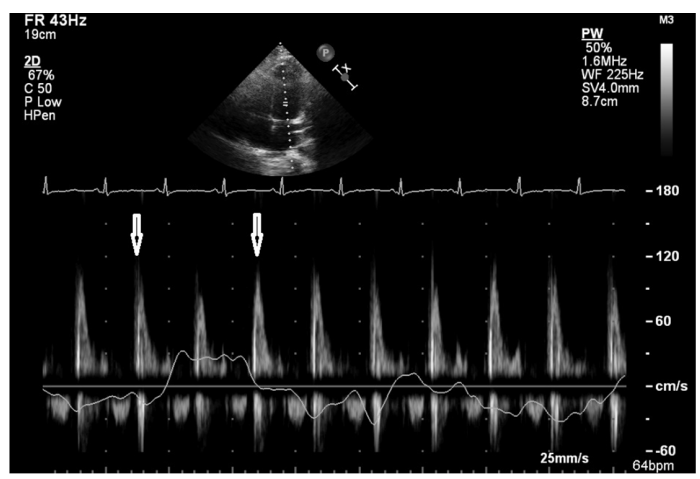

Figure 1. Pulsed-Wave Spectral Doppler echocardiographic image in apical four-chamber view showing no fall of the mitral inflow velocity (E) following inspiration. One third of patients might not have this respiratory change. ${ }^{[5]}$ The E/A ratio is $>1$, and demonstrates no abnormality of relaxation patterns. Because of elevated filling pressures, mitral and tricuspid inflow velocities can be pseudo-normalized

In addition, there may be evidence of pericardial thickening in M-mode with the presence of parallel epicardial and pericardial tracings (also known as "railroad tracks"). ${ }^{[6]} 2-\mathrm{D}$ findings include dilatation of the IVC without inspiratory collapse and septal bounce of the interventricular septum. ${ }^{[7]}$ Doppler echo demonstrates a fall of the mitral inflow velocity from $25 \%-40 \%$ and tricuspid velocity of $>40 \%$ in the cardiac cycle immediately following respiration, and hepatic vein flow reversal which is the most-specific echocardiographic finding for constriction. ${ }^{[1]}$ Tissue Doppler demonstrates the presence of annulus reversus (a medial annular e' to lateral annular e' ratio $>1$ which is a reversal of the normal lateral/medial ratio) and annulus paradoxus. None of these findings are diagnostic individually, and absence does not preclude diagnosis. Our patient had none of the findings save for a dilated IVC, which is a nonspecific finding and was nondiagnostic in our patient until cardiac catheterization. Our patient certainly had hemodynamic changes and 
ascites that rapidly corrected following pericardiectomy, yet none of these hemodynamic changes were manifested on echocardiography, as demonstrated in Figures 1 and 2.

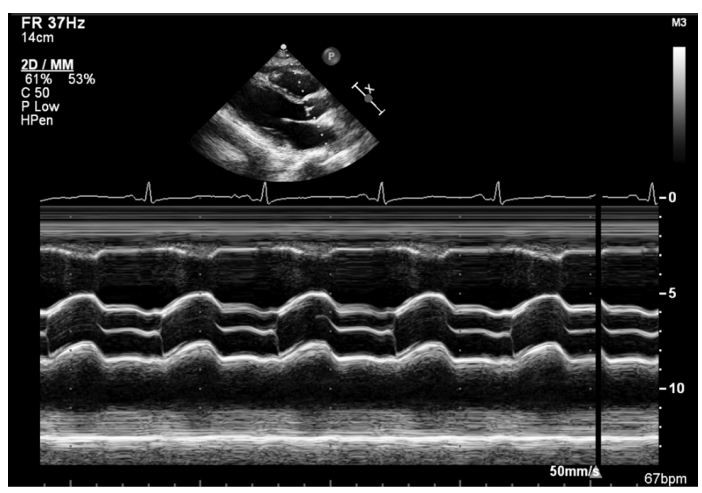

Figure 2. M-mode echocardiographic image in parasternal long-axis showing no septal bounce characteristic of diastolic interventricular septal bounce or shudder

There may be several etiologies for $\mathrm{CP}$ presenting without echocardiographic findings. During the initial stages of constriction, the patient may have a reduction in cardiac output but without significant elevation of filling pressures. ${ }^{[1]} \mathrm{A}$ second possibility is that the patient may be volume depleted, as many of patients with constrictive pericarditis who are on diuretics may be. Echocardiographically covert CP may be uncovered by a rapid volume challenge which restores the elevated filling pressures. ${ }^{[8]}$ A third etiology results from the variation in the location of pericardial constriction. Although most pericardial constriction presents over the entire pericardium or over the right sided chambers, it may also present with atypical presentations when present focally over the pericardium, such as over the ascending aorta, pulmonary trunk, or the mid-ventricle. ${ }^{[9,10]}$

This case suggests that the severity of the constriction as well as its symptomatology does not necessarily correlate to echocardiographic findings. It also underscores that importance of using imaging based on structural changes concomitantly with those that manifest the hemodynamic changes present with $\mathrm{CP}$, and approaching suspected $\mathrm{CP}$ with a holistic approach; this includes usage of newer echocardiographic applications such as strain imaging, as well as CT, cardiac MRI, and cardiac catheterization. The Mayo Clinic diagnostic algorithm for the diagnosis of $\mathrm{CP}$ requires the presence of a mitral inflow $\mathrm{E} / \mathrm{A}$ ratio $>0.8$ and a dilated inferior vena cava for CP to be likely. If present, then ventricular septal motion abnormality with respiration is considered, but if not present, then other non-echocardiographic modalities are utilized. ${ }^{[1]}$ The usage of this criteria in our patient was particularly apt and stressed the correct diagnosis, suggesting good sensitivity for the criteria.

\section{CONFLicts of InTEREST Disclosure}

The authors have no competing interests to declare.

\section{REFERENCES}

[1] Syed FF, Schaff HV, Oh JK. Constrictive pericarditis-a curable diastolic heart failure. Nature Reviews Cardiology. 2014; 11: 530544. PMid:25072910 https://doi.org/10.1038/nrcardio.2 014.100

[2] Adler Y, Charron P, Imazio M, et al. 2015 ESC Guidelines for the diagnosis and management of pericardial diseases: The Task Force for the Diagnosis and Management of Pericardial Diseases of the European Society of Cardiology (ESC) Endorsed by: The European Association for Cardio-Thoracic Surgery (EACTS). Eur Heart J. 2015; 36: 2921. PMid:26320112 https://doi.org/10.1093/ eurheartj/ehv318

[3] Boonyaratavej S, Oh JK, Tajik AJ, et al. Comparison of mitral inflow and superior vena cava Doppler velocities in chronic obstructive pulmonary disease and constrictive pericarditis. J Am Coll Cardiol. 1998; 32(7): 2043-8. https://doi.org/10.1016/S0735-1097(98)0 0472-0

[4] Tei C, Child JS, Tanaka H, et al. Atrial systolic notch on the interventricular septal echogram: an echocardiographic sign of constrictive pericarditis. J Am Coll Cardiol. 1983; 1(3): 907-12. https://doi.org/10.1016/S0735-1097 (83)80207-1
[5] Welch TD, Ling H, Espinosa RE, et al. Echocardiographic diagnosis of constrictive pericarditis: Mayo Clinic Criteria. Circ. Cardiovasc. Imaging. 2014; 7: 526-534. PMid:24633783 https: //doi.org/10.1161/CIRCIMAGING.113.001613

[6] Engel PJ, Fowler NO, Tei C, et al. M-mode echocardiography in constrictive pericarditis. J Am Coll Cardiol. 1985; 6(2): 471-4. https ://doi .org/10.1016/S0735-1097 (85) 80188-1

[7] Hoit BD. Imaging the pericardium. Cardiol Clin. 1990; 8(4): 587-600. Review. PMid:2249213

[8] Bush CA, Stang JM, Wooley CF, et al. Occult Constricive Pericardial Disease: Diagnosis by Rapid Volume Expansion and Correction by Pericardiectomy. Circulation. 1977; 56: 924-930. PMid:923061 https://doi.org/10.1161/01.CIR.56.6.924

[9] Barros JL, Pérez Gómez F. Pulmonary stenosis due to external compression by a pericardial band. Br. Heart J. 1967; 29: 947-949. https://doi.org/10.1136/hrt.29.6.947

[10] Tugcu A, Yildirimturk O, Duran C, et al. Constrictive pericarditis impressing and narrowing the ascending aorta. Echocardiography. 2008; 25: 768-771. PMid:18754936 https://doi.org/10.1111/ j.1540-8175.2008.00660.x 